\title{
The Pricing of Vulnerable Options in a Fractional Brownian Motion Environment
}

\author{
Chao Wang, Shengwu Zhou, and Jingyuan Yang \\ China University of Mining and Technology, Xuzhou 221116, China \\ Correspondence should be addressed to Chao Wang; 172305115@qq.com
}

Received 6 January 2015; Revised 16 June 2015; Accepted 22 June 2015

Academic Editor: Juan R. Torregrosa

Copyright (C) 2015 Chao Wang et al. This is an open access article distributed under the Creative Commons Attribution License, which permits unrestricted use, distribution, and reproduction in any medium, provided the original work is properly cited.

Under the assumption of the stock price, interest rate, and default intensity obeying the stochastic differential equation driven by fractional Brownian motion, the jump-diffusion model is established for the financial market in fractional Brownian motion setting. With the changes of measures, the traditional pricing method is simplified and the general pricing formula is obtained for the European vulnerable option with stochastic interest rate. At the same time, the explicit expression for it comes into being.

\section{Introduction}

Vulnerable option is a kind of option with credit risk that refers to a risk, a borrower that will default on any type of debt by failing to make required payments. Johnson and Stulz [1] firstly substituted default risk into option pricing and advanced a new definition called vulnerable option. Klein [2] obtained the pricing formula for vulnerable option with martingale method. Ammann [3] developed Klein's model on the basis of structural approach. He finally obtained the explicit expression for vulnerable option under the assuming of interest rate and default intensity obeying the stochastic differential equation. What is more, other academics such as Chang and Hung [4] also discussed this problem, while all the discussions stated above are in the environment of geometric Brownian motion. Because of the inadequacies of geometric Brownian motion in describing the self-similarity and long-term dependence of stock prices, fractional Brownian motion is widely used into asset pricing. Hu and Øksendal [5] developed the structural approach in the condition that the stock prices followed a fractional Brownian motion and they proved that the correspondence to fractional Black-Scholes market had no arbitrage. For more literature on fractional Brownian motion, we can refer to Øksendal [6]. But there is another problem that fractional Black-Scholes market does not have equivalent martingale measure according to
Sottinen and Valkeila [7]. Necula [8] applied quasimartingale method to the risk neutral measure. Huang et al. [9] obtained the explicit expression for the European option price under the assuming of fractional Black-Scholes market. Su and Wang [10] and Li and Ma [11] derived the closed form formula for the price of the vulnerable European option by the method of changing measures.

In this paper, we will use quasi-martingale method to change measures, so we can derive the general pricing formula for the European vulnerable option under the assuming of the stock price obeying the jump-diffusion model, the interest rate and default intensity obeying Vasicek model which are driven by fractional Brownian motion.

\section{Market Environment}

Let the uncertainty in the economy be described by the filtered probability space $\left(\Omega, \mathscr{F}, P,\left(\mathscr{F}_{t}\right)_{0 \leq t \leq T}\right) . r(t)$ is the short-term interest rate which is consistently positive and $\mathscr{F}_{t^{-}}$ measurable in this space. Assume that $Q$ is a risk neutral martingale measure under which the discounted asset price processes are martingales.

Suppose the stock price is given by

$$
d S(t)=r(t) S(t) d t+\sigma_{1} S(t) d B_{H 1}(t)+S(t) d J(t)
$$


where $\sigma_{1}$ is positive constant and $\left\{B_{H}(t), t \geq 0\right\}$ is a fractional Brownian motion whose Hurst parameter is $H(1 / 2 \leq H<1)$ in the space $\left(\Omega, \mathscr{F}, P,\left(\mathscr{F}_{t}\right)_{0 \leq t \leq T}\right) .\{J(t), t \geq 0\}$ is a composite Poisson process, $J(t)=\sum_{i=1}^{N(t)} Y_{i}$, and $Y_{i}$ represents the $i$ th jump range of $J(t)$ (using a convention that $Y_{0}=0$ if there are no jumps). Consider $E\left(Y_{i}\right)=\theta . Y_{i}$ is a sequence of independent identically distributed random variables with the finite expected value. $Y_{i}$ and $B_{H}(t)$ are mutually independent and $Y_{i}$ and $N(t)$ are mutually independent. $\{N(t), t \geq 0\}$ is a Poisson process whose intensity is $\lambda(t) .\{\lambda(t), t \geq 0\}$ is a nonnegative adapted stochastic process which is integrable on any finite time interval. $N(t), \lambda(t)$, and $B_{H}(t)$ are mutually independent.

Suppose that $Y_{i}+1$ follows log-normal distribution $\ln \left(Y_{i}+\right.$ $1) \sim N\left(\mu_{J}, \sigma_{J}^{2}\right)$ so that $\ln (\theta+1)=E\left[\ln \left(Y_{i}+1\right)\right]=\mu_{J}+(1 / 2) \sigma_{J}^{2}$; then we have

$$
\ln \left(Y_{i}+1\right) \sim N\left(\ln (\theta+1)-\frac{1}{2} \sigma_{J}^{2}, \sigma_{J}^{2}\right) .
$$

Suppose that the interest rate and default intensity follow Vasicek model under the risk neutral measure

$$
\begin{aligned}
& d r(t)=\alpha[\beta-r(t)] d t+\sigma_{2} d B_{H 2}(t) \\
& d \lambda(t)=a[b-\lambda(t)] d t+\sigma_{3} d B_{H 3}(t),
\end{aligned}
$$

where $\alpha, \beta, a, b, \sigma_{2}$, and $\sigma_{3}$ are all positive constants. The covariance matrix of $B_{H_{1}}(t), B_{H_{2}}(t), B_{H 3}(t)$ is

$$
\left(\begin{array}{ccc}
1 & \rho_{12} & \rho_{13} \\
\rho_{12} & 1 & \rho_{23} \\
\rho_{13} & \rho_{23} & 1
\end{array}\right) t .
$$
firstly.

In order to prove the theorem, we introduce two lemmas

Lemma 1 (see [8]). We denote by $\widetilde{E_{t}}[\cdot]$ the quasi-conditional expectation with respect to the risk neutral measure. Then the price at every $t \in[0, T]$ of a bounded $F_{T}$-measurable claim $F$ is given by

$$
F_{t}=e^{-r(T-t)} \widetilde{E_{t}}[F]
$$

Lemma 2 (see [12]). If $(X, Y) \sim N(\mu, \Sigma)$, where $\mu=\left(\begin{array}{c}\mu_{X} \\ \mu_{Y}\end{array}\right)$, $\Sigma=\left(\begin{array}{c}\sigma_{X}^{2}, \rho \sigma_{X} \sigma_{Y} \\ \rho \sigma_{X} \sigma_{Y}, \sigma_{Y}^{2}\end{array}\right)$, then

$$
\begin{aligned}
& E\left(e^{X} I_{\{X>b\}}\right) \\
& \quad=\exp \left(\mu_{X}+\frac{\sigma_{X}^{2}}{2}\right) N\left(\frac{-b+\mu_{Y}+\operatorname{cov}(X, Y)}{\sigma_{Y}}\right),
\end{aligned}
$$

where $N(\cdot)$ is a standard normal distribution.

\section{Pricing Options}

In this section, we intend to discuss pricing vulnerable options in a Fractional Brownian Motion Environment.
We define that the default time is $\tau$ and $\omega$ represents the recovery rate due to the bankruptcy or reorganization, where $\omega$ is a constant. When the writer of the option defaults, the payoff is given by $\omega$ times the payoff of the default-free option at maturity. The price at every $t \in[0, T]$ of an European vulnerable call option with strike price $K$ and maturity $T$ is given by

$$
\begin{aligned}
& C(t, T)=E\left[e ^ { - \int _ { t } ^ { T } r ( u ) d u } \left(\omega\left(S_{T}-K\right)^{+} I_{\{\tau \leq T\}}\right.\right. \\
& \left.\left.\quad+\left(S_{T}-K\right)^{+} I_{\{\tau>T\}}\right) \mid \mathscr{F}_{t}\right] .
\end{aligned}
$$

Note that $I_{\{\tau \leq T\}}+I_{\{\tau>T\}}=1$; we have

$$
\begin{aligned}
C(t, T) & \\
= & \omega E\left[e^{-\int_{t}^{T} r(u) d u}\left(S_{T}-K\right)^{+} \mid \mathscr{F}_{t}\right] \\
& +(1-\omega) E\left[e^{-\int_{t}^{T} r(u) d u}\left(S_{T}-K\right)^{+} I_{\{\tau>T\}} \mid \mathscr{F}_{t}\right] .
\end{aligned}
$$

Since $\left(\mathscr{F}_{t}\right)_{0 \leq t \leq T}$ is a filtration, then $\mathscr{F}_{t} \subseteq \mathscr{F}_{T}$. Suppose there is no default at present time, by the law of iterated conditional expectations; therefore,

$$
\begin{aligned}
E & {\left[e^{-\int_{t}^{T} r(u) d u}\left(S_{T}-K\right)^{+} I_{\{\tau>T\}} \mid \mathscr{F}_{t}\right] } \\
& =E\left[E\left[e^{-\int_{t}^{T} r(u) d u}\left(S_{T}-K\right)^{+} I_{\{\tau>T\}} \mid \mathscr{F}_{t}\right] \mid \mathscr{F}_{T}\right] .
\end{aligned}
$$

Obviously $e^{-\int_{t}^{T} r(u) d u}\left(S_{T}-K\right)^{+} I_{\{\tau>T\}}$ is bonded and absolutely integrable, so we can interchange the two expectations by Fubini's theorem

$$
\begin{aligned}
& E\left[e^{-\int_{t}^{T} r(u) d u}\left(S_{T}-K\right)^{+} I_{\{\tau>T\}} \mid \mathscr{F}_{t}\right] \\
& \quad=E\left[e^{-\int_{t}^{T} r(u) d u}\left(S_{T}-K\right)^{+} E\left[I_{\{\tau>T\}} \mid \mathscr{F}_{T}\right] \mid \mathscr{F}_{t}\right] .
\end{aligned}
$$

Since the full path of $\lambda(t)$ is known at time $T$, we have [13]

$$
E\left[I_{\{\tau>T\}} \mid \mathscr{F}_{T}\right]=P(\tau>T)=e^{-\int_{t}^{T} \lambda(u) d u} .
$$

Then (9) can be written as

$$
\begin{aligned}
C(t, T) & \\
= & \omega E\left[e^{-\int_{t}^{T} r(u) d u}\left(S_{T}-K\right)^{+} \mid \mathscr{F}_{t}\right] \\
& +(1-\omega) E\left[e^{-\int_{t}^{T}[r(u)+\lambda(u)] d u}\left(S_{T}-K\right)^{+} \mid \mathscr{F}_{t}\right] .
\end{aligned}
$$

For convenience, let $C(t, T)=I+I I$, where $I=$ $\omega E\left[e^{-\int_{t}^{T} r(u) d u}\left(S_{T}-K\right)^{+} \mid \mathscr{F}_{t}\right]$ and $I I=(1-\omega) E\left[e^{-\int_{t}^{T}[r(u)+\lambda(u)] d u}\right.$ $\left.\left(S_{T}-K\right)^{+} \mid \mathscr{F}_{t}\right] ; I$ and $I I$ are given as follows. 
Theorem 3. Consider

$$
\begin{aligned}
I= & \omega \sum_{n=0}^{\infty} \frac{\lambda(t)(T-t)^{n} e^{-\lambda(t)(T-t)}}{n !} \\
& \cdot\left[(1+\theta)^{n} S_{t} N\left(d_{1}(n)\right)+K P(t, T) N\left(d_{2}(n)\right)\right],
\end{aligned}
$$

where

$$
\begin{aligned}
& d_{2}(n) \\
& =\frac{-\ln \left(K / S_{t}\right)+\Delta(t, T)+\Pi(t, T)+\ln (1+\theta)^{n}-n \sigma_{J}^{2} / 2}{\sqrt{n \sigma_{J}^{2}+2 H \int_{t}^{T} \sigma^{*}(u, T)^{2} u^{2 H-1} d u}} \\
& d_{1}(n)=d_{2}(n)+\sqrt{n \sigma_{J}^{2}+2 H \int_{t}^{T} \sigma^{*}(u, T)^{2} u^{2 H-1} d u} \\
& \sigma^{*}(u, T)^{2}=\sigma_{2}^{2} M(u, T, \alpha)^{2}+\sigma_{1}^{2}+2 \rho_{12} \sigma_{1} \sigma_{2} M(u, T, \alpha) \\
& P(t, T)=\exp \{-\beta(T-t)-[r(t)-\beta] M(t, T, \alpha) \\
& \left.\quad+H \sigma_{2}^{2} \int_{t}^{T} M(u, T, \alpha)^{2} u^{2 H-1} d u\right\} \\
& \Delta(t, T)=\beta(T-t)+[r(t)-\beta] M(t, T, \alpha)-\frac{1}{2} \sigma_{1}^{2}\left(T^{2 H}\right. \\
& \left.\quad-t^{2 H}\right) \\
& \Pi(t, T)=-2 H \int_{t}^{T}\left[\sigma_{2}^{2} M(u, T, \alpha)^{2}+\rho_{12} \sigma_{1} \sigma_{2} M(u, T, \alpha)\right] \\
& \quad \cdot u^{2 H-1} d u \\
& M(t, T, \alpha)=\frac{1}{\alpha}\left(1-e^{-\alpha(T-t)}\right) .
\end{aligned}
$$

Proof. By means of Lemma 1 we can see that the discounted asset price process whose numeraire is $F(t)$ is a quasimartingale. So we can define different equivalent quasimartingale measures for different numeraires.

Suppose there is a bank account $B_{t}=\exp \left\{\int_{0}^{t} r(u) d u\right\}$ and a coupon with maturity $T$. The price at every $t \in[0, T]$ of the coupon is $P(t, T)$. Then the forward quasi-martingale measure $Q^{T}$ equivalent to $Q$ by the Radon-Nikodym derivative is given as

$$
\frac{d Q^{T}}{d Q}=\frac{P(T, T)}{P(0, T) B_{T}}=\frac{1}{P(0, T) B_{T}}
$$

Since

$$
P(t, T)=E\left[e^{-\int_{t}^{T} r(u) d u} \mid \mathscr{F}_{t}\right]
$$

hence

$$
\frac{d Q^{T}}{d Q}=\frac{e^{-\int_{0}^{T} r(u) d u}}{E\left[e^{-\int_{0}^{T} r(u) d u} \mid \mathscr{F}_{t}\right]}
$$

Using (3) we can have the expression for $r(t)$ [14]

$$
\begin{gathered}
r(t)=e^{-\alpha(t-s)} r(s)+\beta\left(1-e^{-\alpha(t-s)}\right) \\
+\sigma_{2} \int_{s}^{t} e^{-\alpha(t-u)} d B_{H 2}(u) .
\end{gathered}
$$

We integrate two sides from $[t, T]$ exclusively:

$$
\begin{aligned}
\int_{t}^{T} r(s) d s= & \beta(T-t)+[r(t)-\beta] \int_{t}^{T} e^{-\alpha(s-t)} d s \\
& +\int_{t}^{T} \sigma_{2} \int_{t}^{s} e^{-\alpha(s-u)} d B_{H 2}(u) d s \\
= & \beta(T-t)+\frac{1}{\alpha}[r(t)-\beta]\left(1-e^{-\alpha(T-t)}\right) \\
& +\sigma_{2} \int_{t}^{T} \int_{u}^{T} e^{-\alpha(s-u)} d s d B_{H 2}(u) .
\end{aligned}
$$

Using Fubini's theorem [15], we exchange the last item's integral sequence and obtain

$$
\begin{aligned}
\int_{t}^{T} r(s) d s= & \beta(T-t)+\frac{1}{\alpha}[r(t)-\beta]\left(1-e^{-\alpha(T-t)}\right) \\
& +\sigma_{2} \int_{t}^{T} \int_{t}^{s} e^{-\alpha(s-u)} d B_{H 2}(u) d s \\
= & \beta(T-t)+\frac{1}{\alpha}[r(t)-\beta]\left(1-e^{-\alpha(T-t)}\right) \\
& +\frac{1}{\alpha} \sigma_{2} \int_{t}^{T}\left(1-e^{-\alpha(T-u)}\right) d B_{H 2}(u) .
\end{aligned}
$$

Let $M(t, T, \alpha)=(1 / \alpha)\left(1-e^{-\alpha(T-t)}\right)$, using multidimensional fractional Itô's lemma [16]

$$
\begin{aligned}
& P(t, T)=E\left[e^{-\int_{t}^{T} r(u) d u} \mid \mathscr{F}_{t}\right]=E[\exp \{-\beta(T-t) \\
& \left.\left.\quad-[r(t)-\beta] M(t, T, \alpha)+\sigma_{2} \int_{t}^{T}-M(u, T, \alpha) d B_{H 2}(u)\right\}\right]=\exp \{-\beta(T-t) \\
& \quad-[r(t)-\beta] M(t, T, \alpha) \\
& \left.\quad+H \sigma_{2}^{2} \int_{t}^{T} M(u, T, \alpha)^{2} u^{2 H-1} d u\right\} .
\end{aligned}
$$

So the Radon-Nikodym derivative is

$$
\begin{aligned}
\frac{d Q^{T}}{d Q} & =\frac{e^{-\int_{0}^{T} r(u) d u}}{E\left[e^{-\int_{0}^{T} r(u) d u} \mid \mathscr{F}_{t}\right]} \\
& =e^{-\sigma_{2} \int_{0}^{T} M(u, T, \alpha) d B_{H 2}(u)-H \sigma_{2}^{2} \int_{0}^{T} M(u, T, \alpha)^{2} u^{2 H-1} d u} .
\end{aligned}
$$

Using fractional Girsanov's theorem [14]

$$
\begin{aligned}
& B_{H 2}^{T}(u)=B_{H 2}(u)+2 H \sigma_{2} \int_{0}^{t} M(u, T, \alpha) u^{2 H-1} d u \\
& B_{H 1}^{T}(u)=B_{H 1}(u)+2 H \rho_{12} \sigma_{2} \int_{0}^{t} M(u, T, \alpha) u^{2 H-1} d u .
\end{aligned}
$$


$B_{H 1}^{T}(u)$ and $B_{H 2}^{T}(u)$ are fractional Brownian motions under measure $Q^{T}$.

So, we can calculate $I=\omega E^{T}\left[P(t, T)\left(S_{T}-K\right)^{+} \mid \mathscr{F}_{t}\right]$ under measure $Q^{T}$.

Since

$$
\begin{aligned}
E^{T}[ & \left.P(t, T)\left(S_{T}-K\right)^{+} \mid \mathscr{F}_{t}\right] \\
= & E^{T}\left[P(t, T) S_{T} I_{\left\{S_{T} \geq K\right\}} \mid \mathscr{F}_{t}\right] \\
& -K E^{f}\left[P(t, T) I_{\left\{S_{T} \geq K\right\}} \mid \mathscr{F}_{t}\right] .
\end{aligned}
$$

$$
S_{T}=S_{t} e^{\beta(T-t)+[r(t)-\beta] M(t, T, \alpha)+\sigma_{2} \int_{t}^{T} M(u, T, \alpha) d B_{H 2}(u)-(1 / 2) \sigma_{1}^{2}\left(T^{2 H}-t^{2 H}\right)+\int_{t}^{T} \sigma_{1} d B_{H 1}(u)+\sum_{i=0}^{N_{t}} \ln \left(Y_{i}+1\right)} .
$$

Using fractional Girsanov's theorem, we have the expression for $S_{T}$ under measure $Q^{T}$

$$
\begin{aligned}
S_{T} & =S_{t} \exp \left\{\Delta(t, T)+\sigma_{2} \int_{t}^{T} M(u, T, \alpha) d B_{H 2}(u)\right. \\
& \left.+\int_{t}^{T} \sigma_{1} d B_{H 1}(u)+\sum_{i=0}^{N_{t}} \ln \left(Y_{i}+1\right)\right\} \\
& =S_{t} \exp \left\{\Delta(t, T)+\sigma_{2} \int_{t}^{T} M(u, T, \alpha) d B_{H 2}^{T}(u)\right. \\
& \left.+\int_{t}^{T} \sigma_{1} d B_{H 1}^{T}(u)+\sum_{i=0}^{N_{t}} \ln \left(Y_{i}+1\right)+\Pi(t, T)\right\},
\end{aligned}
$$

where

$\Pi(t, T)$

$$
\begin{aligned}
& =-2 H \int_{t}^{T}\left[\sigma_{2}^{2} M(u, T, \alpha)^{2}+\rho_{12} \sigma_{1} \sigma_{2} M(u, T, \alpha)\right] \\
& \cdot u^{2 H-1} d u .
\end{aligned}
$$

Since $I_{2}=K E\left[P(t, T) I_{\left\{S_{T} \geq K\right\}} \mid \mathscr{F}_{t}\right]=K P(t, T) E^{T}\left[I_{\left\{S_{T} \geq K\right\}}\right.$ $\left.\mid \mathscr{F}_{t}\right]$, we substitute $S_{T}$ into $I_{\left\{S_{T} \geq K\right\}}$; then

$$
\begin{aligned}
& I_{\left\{S_{T} \geq K\right\}} \\
& =I_{\left\{\sigma_{2} \int_{t}^{T} M(u, T, \alpha) d B_{H 2}^{T}(u)+\int_{t}^{T} \sigma_{1} d B_{H 1}^{T}(u)+\sum_{i=0}^{N_{t}} \ln \left(Y_{i}+1\right) \geq \ln \left(K / S_{t}\right)-\Delta(t, T)-\Pi(t, T)\right\}},
\end{aligned}
$$

since

$$
\begin{aligned}
& \sigma_{2} \int_{t}^{T} M(u, T, \alpha) d B_{H 2}^{T}(u) \\
& \quad \sim N\left(0,2 H \sigma_{2}^{2} \int_{t}^{T} M(u, T, \alpha)^{2} u^{2 H-1} d u\right) \\
& \int_{t}^{T} \sigma_{1} d B_{H 1}^{T}(u) \sim N\left(0, \sigma_{1}^{2}\left(T^{2 H}-t^{2 H}\right)\right) .
\end{aligned}
$$

we define $I_{1}=E^{T}\left[P(t, T) S_{T} I_{\left\{S_{T} \geq K\right\}} \mid \mathscr{F}_{t}\right]$ and $I_{2}=K E^{T}$ $\left[P(t, T) I_{\left\{S_{T} \geq K\right\}} \mid \mathscr{F}_{t}\right]$ for convenience. We will calculate $I_{2}$ firstly.

Using (1) we have the expression for $S_{T}$

$$
\begin{aligned}
S_{T} & =S_{t} \exp \left\{\int_{t}^{T} r(u) d u-\frac{1}{2} \sigma_{1}^{2}\left(T^{2 H}-t^{2 H}\right)\right. \\
& \left.+\int_{t}^{T} \sigma_{1} d B_{H 1}(u)+\sum_{i=0}^{N_{t}} \ln \left(Y_{i}+1\right)\right\} .
\end{aligned}
$$

We substitute $\int_{t}^{T} r(u) d u$ into it; then
When $N_{t}=n$,

$$
\sum_{i=0}^{N_{t}} \ln \left(Y_{i}+1\right) \sim N\left(\ln (1+\theta)^{n}-\frac{1}{2} n \sigma_{J}^{2}, n \sigma_{J}^{2}\right)
$$

According to the nature of normal distribution, when $N_{t}=n$, we have

$$
\begin{aligned}
E^{T} & {\left[I_{\left\{S_{T} \geq K\right\}} \mid \mathscr{F}_{t}\right] } \\
& =\sum_{n=0}^{\infty} \frac{\lambda(t)(T-t)^{n} e^{-\lambda(t)(T-t)}}{n !} N\left(d_{2}(n)\right),
\end{aligned}
$$

where

$$
\begin{aligned}
& d_{2}(n) \\
& =\frac{-\ln \left(K / S_{t}\right)+\Delta(t, T)+\Pi(t, T)+\ln (1+\theta)^{n}-n \sigma_{J}^{2} / 2}{\sqrt{n \sigma_{J}^{2}+2 H \int_{t}^{T} \sigma^{*}(u, T)^{2} u^{2 H-1} d u}} \\
& \sigma^{*}(u, T)^{2}=\sigma_{2}^{2} M(u, T, \alpha)^{2}+\sigma_{1}^{2}+2 \rho_{12} \sigma_{1} \sigma_{2} M(u, T, \alpha) .
\end{aligned}
$$

So

$$
I_{2}=K P(t, T) \sum_{n=0}^{\infty} \frac{\lambda(t)(T-t)^{n} e^{-\lambda(t)(T-t)}}{n !} N\left(d_{2}(n)\right)
$$

Then we will calculate $I_{1}$.

Since $I_{1}=E\left[P(t, T) S_{T} I_{\left\{S_{T} \geq K\right\}} \mid \mathscr{F}_{t}\right]=P(t, T) E^{T}\left[S_{T} I_{\left\{S_{T} \geq K\right\}}\right.$ $\left.\mid \mathscr{F}_{t}\right]$, we substitute $S_{T}$ into $I_{\left\{S_{T} \geq K\right\}}$; then

$$
\begin{aligned}
E^{T} & {\left[S_{T} I_{\left\{S_{T} \geq K\right\}} \mid \mathscr{F}_{t}\right] } \\
& =S_{t} e^{\Delta(t, T)+\Pi(t, T)} E^{T}\left[e^{\sigma_{2} \int_{t}^{T} M(u, T, \alpha) d B_{H 2}^{T}(u)+\int_{t}^{T} \sigma_{1} d B_{H 1}^{T}(u)+\sum_{i=0}^{N_{t}} \ln \left(Y_{i}+1\right)}\right. \\
& \left.\cdot I_{\left\{\sigma_{2} \int_{t}^{T} M(u, T, \alpha) d B_{H 2}^{T}(u)+\int_{t}^{T} \sigma_{1} d B_{H 1}^{T}(u)+\sum_{i=0}^{N_{t}} \ln \left(Y_{i}+1\right) \geq \ln \left(K / S_{t}\right)-\Delta(t, T)-\Pi(t, T)\right\}}\right] .
\end{aligned}
$$


Using Lemma 2, when $N_{t}=n$,

$$
\begin{aligned}
E^{T}\left[S_{T} I_{\left\{S_{T} \geq K\right\}} \mid \mathscr{F}_{t}\right]= & S_{t} e^{\Delta(t, T)+\Pi(t, T)+\ln (1+\theta)^{n}+H \sigma_{2}^{2} \int_{t}^{T} M(u, T, \alpha)^{2} u^{2 H-1} d u+(1 / 2) \sigma_{1}^{2}\left(T^{2 H}-t^{2 H}\right)+2 H \rho_{12} \sigma_{1} \sigma_{2} \int_{t}^{T} M(u, T, \alpha) u^{2 H-1} d u} \\
& \cdot \sum_{n=0}^{\infty} \frac{\lambda(t)(T-t)^{n} e^{-\lambda(t)(T-t)}}{n !} N\left(d_{1}(n)\right)
\end{aligned}
$$

where

$$
d_{1}(n)=d_{2}(n)+\sqrt{n \sigma_{J}^{2}+2 H \int_{t}^{T} \sigma^{*}(u, T)^{2} u^{2 H-1} d u}
$$

$$
\begin{aligned}
I_{1} & =P(t, T) E^{T}\left[S_{T} I_{\left\{S_{T} \geq K\right\}} \mid \mathscr{F}_{t}\right]=S_{t} e^{\Delta(t, T)+\Pi(t, T)+\ln (1+\theta)^{n}+H \sigma_{2}^{2} \int_{t}^{T} M(u, T, \alpha)^{2} u^{2 H-1} d u+(1 / 2) \sigma_{1}^{2}\left(T^{2 H}-t^{2 H}\right)+2 H \rho_{12} \sigma_{1} \sigma_{2} \int_{t}^{T} M(u, T, \alpha) u^{2 H-1} d u} \\
& \cdot e^{-\beta(T-t)-[r(t)-\beta] M(t, T, \alpha)+H \sigma_{2}^{2} \int_{t}^{T} M(u, T, \alpha)^{2} u^{2 H-1} d u}=(1+\theta)^{n} S_{t} \sum_{n=0}^{\infty} \frac{\lambda(t)(T-t)^{n} e^{-\lambda(t)(T-t)}}{n !} N\left(d_{1}(n)\right) .
\end{aligned}
$$

Hence, when $N_{t}=n$,

$$
\begin{aligned}
I= & \omega\left(I_{1}+I_{2}\right)=\omega \sum_{n=0}^{\infty} \frac{\lambda(t)(T-t)^{n} e^{-\lambda(t)(T-t)}}{n !} \\
& \cdot\left[(1+\theta)^{n} S_{t} N\left(d_{1}(n)\right)+K P(t, T) N\left(d_{2}(n)\right)\right] .
\end{aligned}
$$

\section{Theorem 4. Consider}

$$
\begin{aligned}
I I= & (1-\omega) \sum_{n=0}^{\infty} \frac{\lambda(t)(T-t)^{n} e^{-\lambda(t)(T-t)}}{n !} X(t, T) \\
& \cdot\left(Y(t, T) N\left(d_{3}(n)\right)-K N\left(d_{4}(n)\right)\right),
\end{aligned}
$$

where

$$
\begin{aligned}
& d_{4}(n)=\frac{-\ln \left(K / S_{t}\right)+\Delta(t, T)+\Lambda(t, T)+\ln (1+\theta)^{n}-n \sigma_{J}^{2} / 2}{\sqrt{n \sigma_{J}^{2}+2 H \int_{t}^{T} \sigma^{*}(u, T)^{2} u^{2 H-1} d u}}, \\
& d_{3}(n)=d_{4}(n)+\sqrt{n \sigma_{J}^{2}+2 H \int_{t}^{T} \sigma^{*}(u, T)^{2} u^{2 H-1} d u,} \\
& X(t, T)=\exp \{-(\beta+b)(T-t)-[r(t)-\beta] M(t, T, \alpha)-[r(t)-b] M(t, T, a) \\
& \left.+H \int_{t}^{T}\left[\sigma_{2}^{2} M^{2}(u, T, \alpha)+\sigma_{3}^{2} M^{2}(u, T, a)+2 \rho_{23} \sigma_{2} \sigma_{3} M(u, T, \alpha) M(u, T, a)\right] u^{2 H-1} d u\right\}, \\
& Y(t, T)=S_{t} \exp \left\{\Delta(t, T)+\Lambda(t, T)+\ln (1+\theta)^{n}+H \int_{t}^{T} \sigma^{*}(u, T)^{2} u^{2 H-1} d u\right\}, \\
& \Lambda(t, T)=-2 H \int_{t}^{T}\left[\sigma_{2} M(u, T, \alpha) M_{1}(u)+\rho_{12} \sigma_{1} M_{1}(u)+\rho_{13} \sigma_{1} M_{2}(u)\right] u^{2 H-1} d u, \\
& M_{1}(u)=\sigma_{2} M(u, T, \alpha)+\rho_{23} \sigma_{3} M(u, T, a), \\
& M_{2}(u)=\sigma_{3} M(u, T, a)+\rho_{23} \sigma_{2} M(u, T, \alpha), \\
& M(t, T, a)=\frac{1}{a}\left(1-e^{-a(T-t)}\right) .
\end{aligned}
$$


Proof. Using Lemma 1, the quasi-martingale measure $Q^{\lambda}$ equivalent to $Q$ by the Radon-Nikodym derivative is given as

$$
\frac{d Q^{\lambda}}{d Q}=\frac{e^{-\int_{t}^{T}[r(u)+\lambda(u)] d u}}{E\left[e^{-\int_{t}^{T}[r(u)+\lambda(u)] d u}\right]} .
$$

Using (4) we can have the expression for $\lambda(t)$

$$
\begin{aligned}
\lambda(t)= & e^{-a(t-s)} r(s)+b\left(1-e^{-a(t-s)}\right) \\
& +\sigma_{3} \int_{s}^{t} e^{-a(t-u)} d B_{H 3}(u) .
\end{aligned}
$$

Then we have

$$
\begin{aligned}
\int_{t}^{T} \lambda(s) d s= & b(T-t)+\frac{1}{a}[\lambda(t)-b]\left(1-e^{-a(T-t)}\right) \\
& +\frac{1}{a} \sigma_{3} \int_{t}^{T}\left(1-e^{-a(T-u)}\right) d B_{H 3}(u) .
\end{aligned}
$$

Using multidimensional fractional Itô lemma, let $X(t, T)=$ $E\left[e^{-\int_{t}^{T}[r(u)+\lambda(u)] d u} \mid \mathscr{F}_{t}\right]$; then
Let $M(t, T, a)=(1 / a)\left(1-e^{-a(T-t)}\right)$; then

$$
\begin{aligned}
-\int_{t}^{T} & {[r(u)+\lambda(u)] d u } \\
= & -(\beta+b)(T-t)-[r(t)-\beta] M(t, T, \alpha) \\
& -[\lambda(t)-b] M(t, T, a) \\
& -\sigma_{2} \int_{t}^{T} M(u, T, \alpha) d B_{H 2}(u) \\
& -\sigma_{3} \int_{t}^{T} M(u, T, a) d B_{H 3}(u) .
\end{aligned}
$$

$$
\begin{aligned}
& X(t, T)=\exp \{-(\beta+b)(T-t)-[r(t)-\beta] M(t, T, \alpha)-[r(t)-b] M(t, T, a) \\
& \left.\quad+H \int_{t}^{T}\left[\sigma_{2}^{2} \cdot M^{2}(u, T, \alpha)+\sigma_{3}^{2} M^{2}(u, T, a)+2 \rho_{23} \sigma_{2} \sigma_{3} M(u, T, \alpha) M(u, T, a)\right] u^{2 H-1} d u\right\} .
\end{aligned}
$$

$$
\begin{gathered}
\frac{d Q^{\lambda}}{d Q}=\frac{e^{-\int_{t}^{T}[r(u)+\lambda(u)] d u}}{E\left[e^{-\int_{t}^{T}[r(u)+\lambda(u)] d u}\right]}=\exp \left\{-\sigma_{2} \int_{t}^{T} M(u, T, \alpha) d B_{H 2}(u)-\sigma_{3} \int_{t}^{T} M(u, T, a) d B_{H 3}(u)\right. \\
\left.-H \int_{t}^{T}\left[\sigma_{2}^{2} M^{2}(u, T, \alpha)+\sigma_{3}^{2} M^{2}(u, T, a)+2 \rho_{23} \sigma_{2} \sigma_{3} M(u, T, \alpha) M(u, T, a)\right] u^{2 H-1} d u\right\} .
\end{gathered}
$$


Then we can calculate $I I=(1-\omega) X(t, T) E^{\lambda}\left[\left(S_{T}-K\right)^{+} \mid \mathscr{F}_{t}\right]$ under $Q^{\lambda}$.

Since

$$
\begin{aligned}
E^{\lambda}\left[\left(S_{T}-K\right)^{+} \mid \mathscr{F}_{t}\right]= & E^{\lambda}\left[S_{T} I_{\left\{S_{T} \geq K\right\}} \mid \mathscr{F}_{t}\right] \\
& -K E^{\lambda}\left[I_{\left\{S_{T} \geq K\right\}} \mid \mathscr{F}_{t}\right],
\end{aligned}
$$

we define $I_{3}=E^{\lambda}\left[S_{T} I_{\left\{S_{T} \geq K\right\}} \mid \mathscr{F}_{t}\right]$ and $I_{4}=K E^{\lambda}\left[I_{\left\{S_{T} \geq K\right\}}\right.$ $\left[\mathscr{F}_{t}\right]$ for convenience. We will calculate $I_{4}$ firstly.

Using fractional Girsanov's theorem, we have the expression for $S_{T}$ under measure $Q^{\lambda}$

$$
\begin{aligned}
& S_{T} \\
& =S_{t} e^{\Delta(t, T)+\sigma_{2} \int_{t}^{T} M(u, T, \alpha) d B_{H 2}^{\lambda}(u)+\int_{t}^{T} \sigma_{1} d B_{H 1}^{\lambda}(u)+\sum_{i=0}^{N_{t}} \ln \left(Y_{i}+1\right)+\Pi(t, T)},
\end{aligned}
$$

where

$$
\begin{gathered}
\Delta(t, T)=\beta(T-t)+[r(t)-\beta] M(t, T, \alpha)-\frac{1}{2} \\
\cdot \sigma_{1}^{2}\left(T^{2 H}-t^{2 H}\right) \\
\Lambda(t, T)=-2 H \int_{t}^{T}\left[\sigma_{2} M(u, T, \alpha) M_{1}(u)\right. \\
\left.\quad+\rho_{12} \sigma_{1} M_{1}(u)+\rho_{13} \sigma_{1} M_{2}(u)\right] u^{2 H-1} d u .
\end{gathered}
$$

$$
\begin{aligned}
E^{\lambda}\left[S_{T} I_{\left\{S_{T} \geq K\right\}} \mid \mathscr{F}_{t}\right]= & S_{t} e^{\Delta(t, T)+\Lambda(t, T)} e^{\ln (1+\theta)^{n}-(1 / 2) n \sigma_{J}^{2}+H \sigma_{2}^{2} \int_{t}^{T} M(u, T, \alpha)^{2} u^{2 H-1} d u+(1 / 2) \sigma_{1}^{2}\left(T^{2 H}-t^{2 H}\right)+2 H \rho_{12} \sigma_{1} \sigma_{2} \int_{t}^{T} M(u, T, \alpha) u^{2 H-1} d u+(1 / 2) n \sigma_{J}^{2}} \\
& \cdot \sum_{n=0}^{\infty} \frac{\lambda(t)(T-t)^{n} e^{-\lambda(t)(T-t)}}{n !} N\left(d_{3}(n)\right) .
\end{aligned}
$$

Let

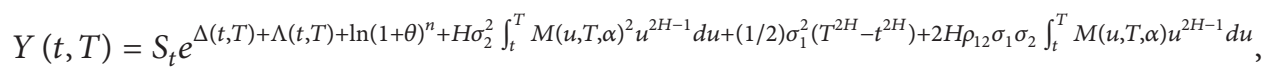

and then

$$
\begin{aligned}
E^{\lambda} & {\left[S_{T} I_{\left\{S_{T} \geq K\right\}} \mid \mathscr{F}_{t}\right] } \\
& =Y(t, T) \sum_{n=0}^{\infty} \frac{\lambda(t)(T-t)^{n} e^{-\lambda(t)(T-t)}}{n !} N\left(d_{3}(n)\right),
\end{aligned}
$$

where

$$
d_{3}(n)=d_{4}(n)+\sqrt{n \sigma_{J}^{2}+2 H \int_{t}^{T} \sigma^{*}(u, T)^{2} u^{2 H-1} d u} .
$$

So

$$
\begin{aligned}
I_{3} & =E^{\lambda}\left[S_{T} I_{\left\{S_{T} \geq K\right\}} \mid \mathscr{F}_{t}\right] \\
& =Y(t, T) \sum_{n=0}^{\infty} \frac{\lambda(t)(T-t)^{n} e^{-\lambda(t)(T-t)}}{n !} N\left(d_{3}(n)\right) .
\end{aligned}
$$

Hence

$$
I I=(1-\omega) X(t, T)\left(I_{3}-I_{4}\right)=(1-\omega)
$$




$$
\begin{aligned}
& \cdot \sum_{n=0}^{\infty} \frac{\lambda(t)(T-t)^{n} e^{-\lambda(t)(T-t)}}{n !} X(t, T) \\
& \cdot\left[Y(t, T) N\left(d_{3}(n)\right)-K N\left(d_{4}(n)\right)\right] .
\end{aligned}
$$

Theorem 5. The price at time t of the European vulnerable call option is

$$
\begin{aligned}
C( & t, T)=\sum_{n=0}^{\infty} \frac{\lambda(t)(T-t)^{n} e^{-\lambda(t)(T-t)}}{n !} \\
& \cdot\left\{\omega\left[(1+\theta)^{n} S_{t} N\left(d_{1}(n)\right)+K P(t, T) N\left(d_{2}(n)\right)\right]\right. \\
& +(1-\omega) X(t, T) \\
& \left.\cdot\left[Y(t, T) N\left(d_{3}(n)\right)-K N\left(d_{4}(n)\right)\right]\right\} .
\end{aligned}
$$

Note 1. When there is no jump process, $H=1 / 2$, and recovery rate is $1,(65)$ can be simplified as

$$
C(t, T)=S_{t} N\left(d_{1}\right)+K P(t, T) N\left(d_{2}\right) .
$$

\section{Numerical Experiments}

In this section, we mainly discuss the influence of different parameters on option prices. Figures are about the relationship between option prices and strike prices with different parameters.

The values of different parameters are as follows:

$$
\begin{aligned}
\alpha & =0.3 ; \\
\beta & =0.05 ; \\
a & =0.2 ; \\
b & =0.02 ; \\
\sigma_{1} & =0.2 ; \\
\sigma_{2} & =0.15 ; \\
\sigma_{3} & =0.25 ; \\
\sigma_{J} & =0.1 ; \\
r(0) & =0.02 ; \\
\lambda(0) & =0.5 ; \\
S(0) & =100 ; \\
T & =1 .
\end{aligned}
$$

For all figures, the horizontal axis shows strike price and the vertical axis shows option value.

Figure 1 is about the influence of Hurst parameter $H$ on option value. As we can see from the figure, the influence will become larger with the increasing of strike prices within limits.

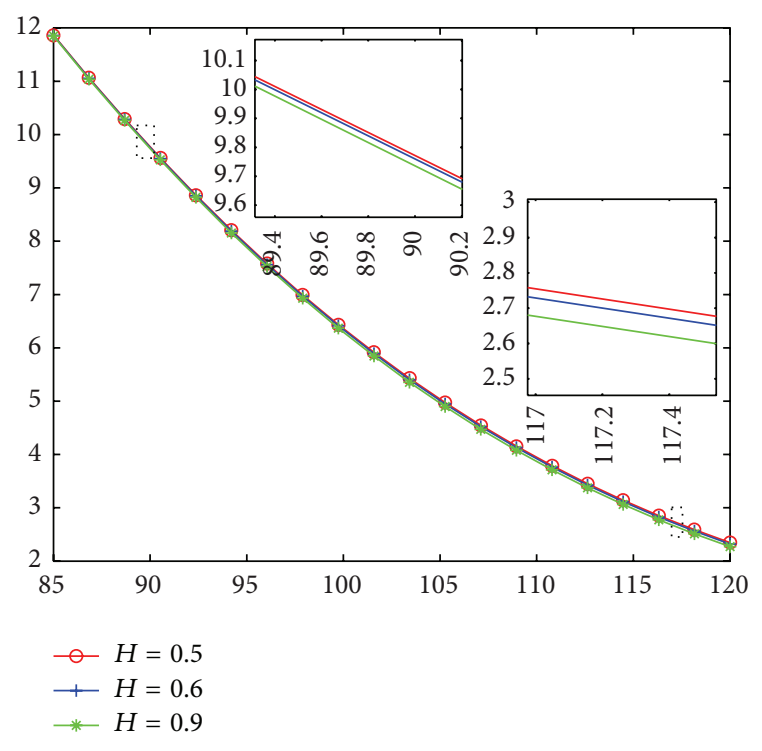

FIgURE 1: The influence of Hurst parameter $H$.

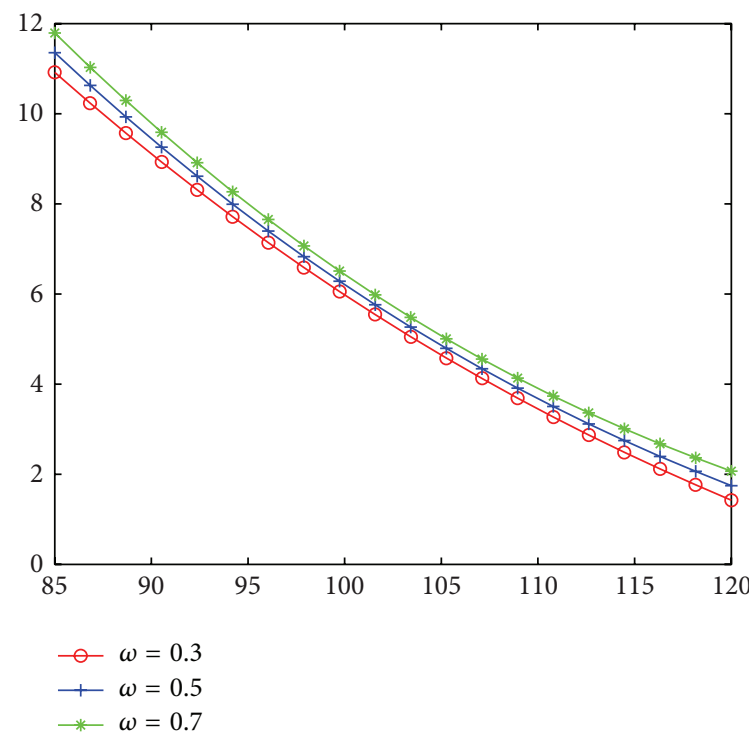

FIGURE 2: The influence of recovery rate $\omega$.

Figure 2 is about the influence of recovery rate $\omega$ on option value. The default risk will decline with the increasing of recovery rate. So the option prices will fall down.

Figures 3, 4, and 5 are about the influences of different covariance. According to these figures, we can see that different covariance has different influences on option value.

\section{Conclusion}

The method of changing measures is widely used for pricing options. In this paper, we develop this method and prove its feasibility in pricing options under the assumption of fractional Brownian motion. What is more, we also take jump process into consideration and obtain the general pricing 


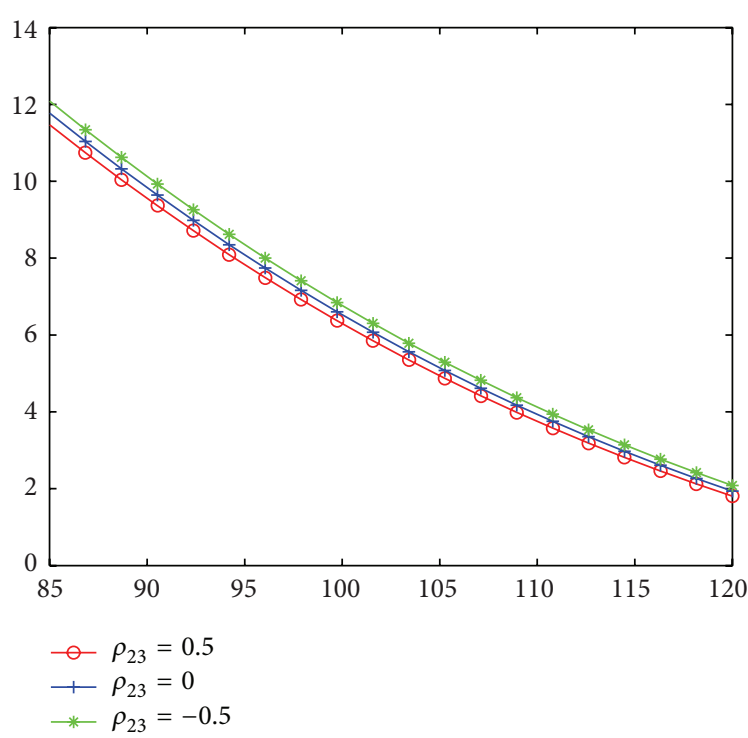

FIgURE 3: The influence of default covariance $\rho_{23}$.

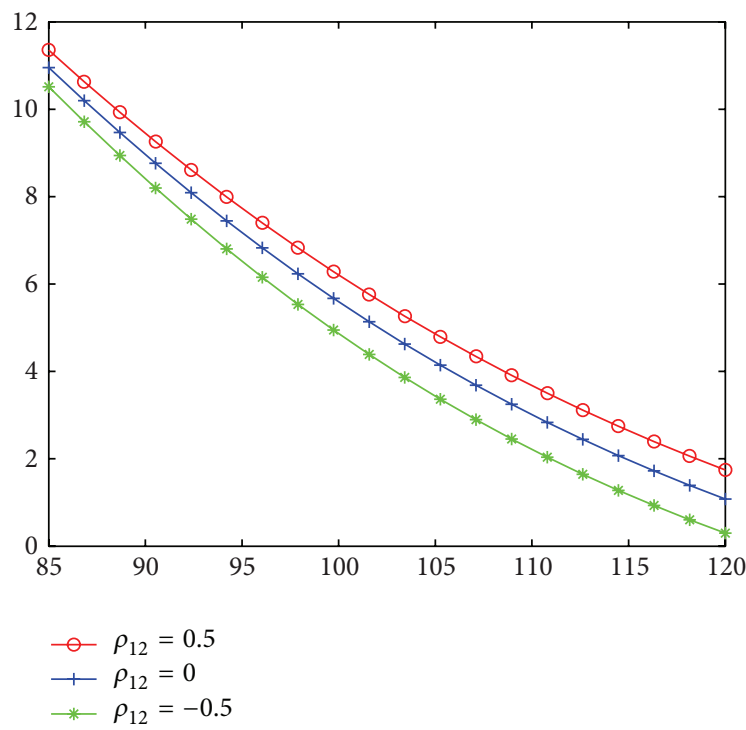

FIGURE 4: The influence of covariance $\rho_{12}$.

formula for the European vulnerable option. Finally, we verify its accuracy through the numerical experiments.

\section{Conflict of Interests}

The authors declare that there is no conflict of interests regarding the publication of this paper.

\section{Acknowledgment}

This work is supported by the Fundamental Research Funds for the Central Universities (2013XK03).

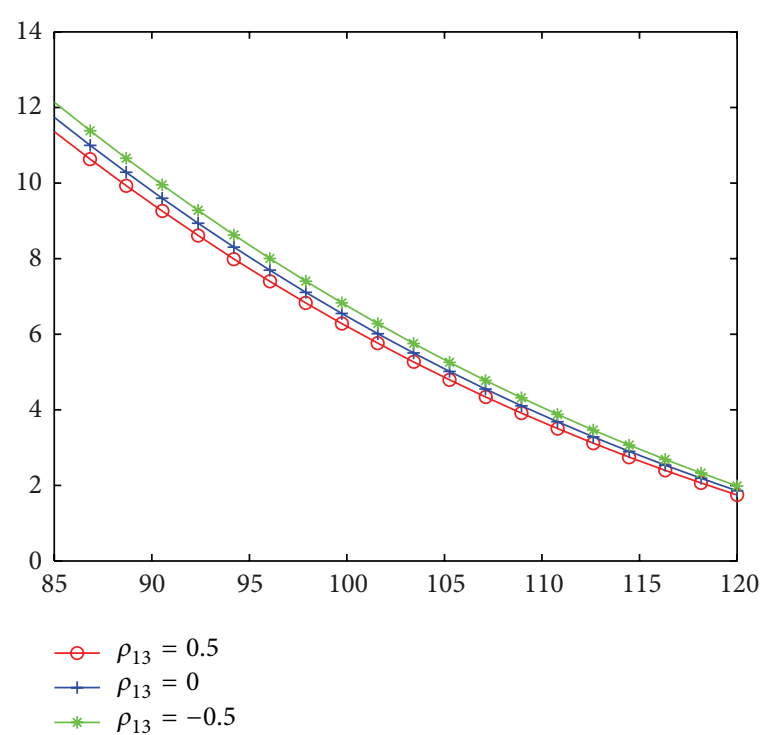

FIGURE 5: The influence of covariance $\rho_{13}$.

\section{References}

[1] H. Johnson and R. Stulz, "The pricing of options with default ris," The Journal of Finance, vol. 42, no. 2, pp. 267-280, 1987.

[2] P. Klein, "Pricing black-scholes options with correlated credit risk," Journal of Banking and Finance, vol. 20, no. 7, pp. 12111229, 1996.

[3] M. Ammann, Credit Risk Valuation: Methods, Models, and Applications, Springer Science \& Business Media, 2001.

[4] L.-F. Chang and M.-W. Hung, "Valuation of vulnerable American options with correlated credit risk," Review of Derivatives Research, vol. 9, no. 2, pp. 137-165, 2006.

[5] Y. Hu and B. Øksendal, "Fractional white noise calculus and applications to finance," Infinite Dimensional Analysis, Quantum Probability and Related Topics, vol. 6, no. 1, pp. 1-32, 2003.

[6] B. Øksendal, Fractional Brownian Motion in Finance, University of Oslo, Oslo, Norway, 2004.

[7] T. Sottinen and E. Valkeila, Fractional Brownian motion as a model in finance, 2001.

[8] C. Necula, Option Pricing in a Fractional Brownian Motion Environment, Bucharest University of Economics, Bucuresti, Romania, 2002.

[9] W. L. Huang, X. X. Tao, and S. H. Li, "Pricing formulae for European options under the fractional Vasicek interest rate model," Acta Mathematica Sinica. Chinese Series, vol. 55, no. 2, pp. 219-230, 2012.

[10] X. Su and W. Wang, "Pricing options with credit risk in a reduced form model," Journal of the Korean Statistical Society, vol. 41, no. 4, pp. 437-444, 2012.

[11] J. Li and S. Ma, "Pricing options with credit risk in Markovian regime-switching markets," Journal of Applied Mathematics, vol. 2013, Article ID 621371, 9 pages, 2013.

[12] F. C. Klebaner, Introduction to Stochastic Calculus with Applications, Imperial College Press, 2005.

[13] R. A. Jarrow and F. Yu, "Counterparty risk and the pricing of defaultable securities," Journal of Finance, vol. 56, no. 5, pp. 1765-1799, 2001. 
[14] S. E. Shreve, Stochastic Calculus for Finance. 2. Continuous Time Models, Springer, 2004.

[15] F. Biagini, Y. Hu, B. Øksendal, and T. Zhang, Stochastic Calculus for Fractional Brownian Motion and Applications, Springer Science \& Business Media, 2008.

[16] Y. S. Mishura, Stochastic Calculus for Fractional Brownian Motion and Related Processes, Springer, 2007. 


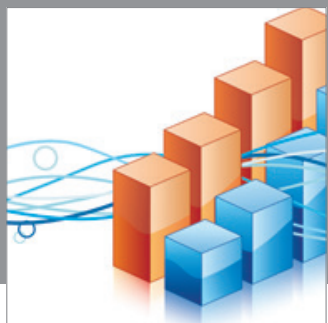

Advances in

Operations Research

mansans

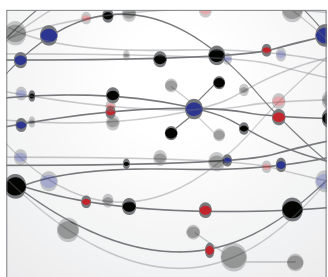

The Scientific World Journal
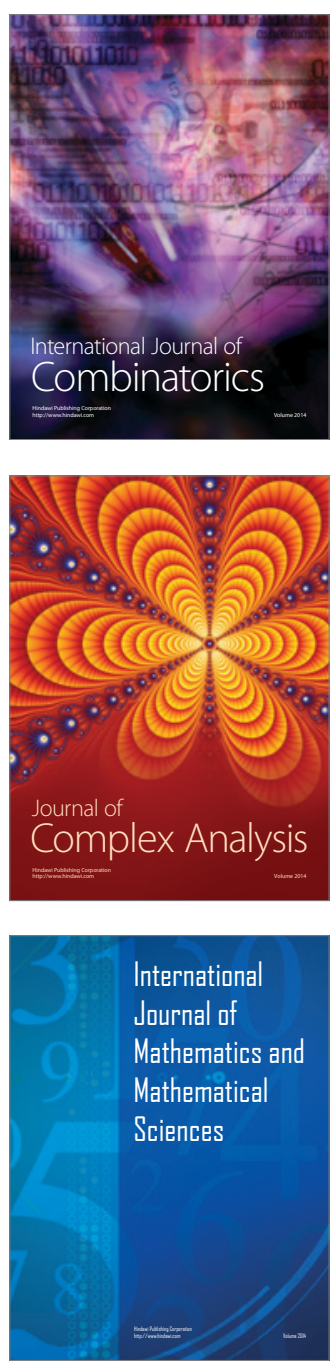
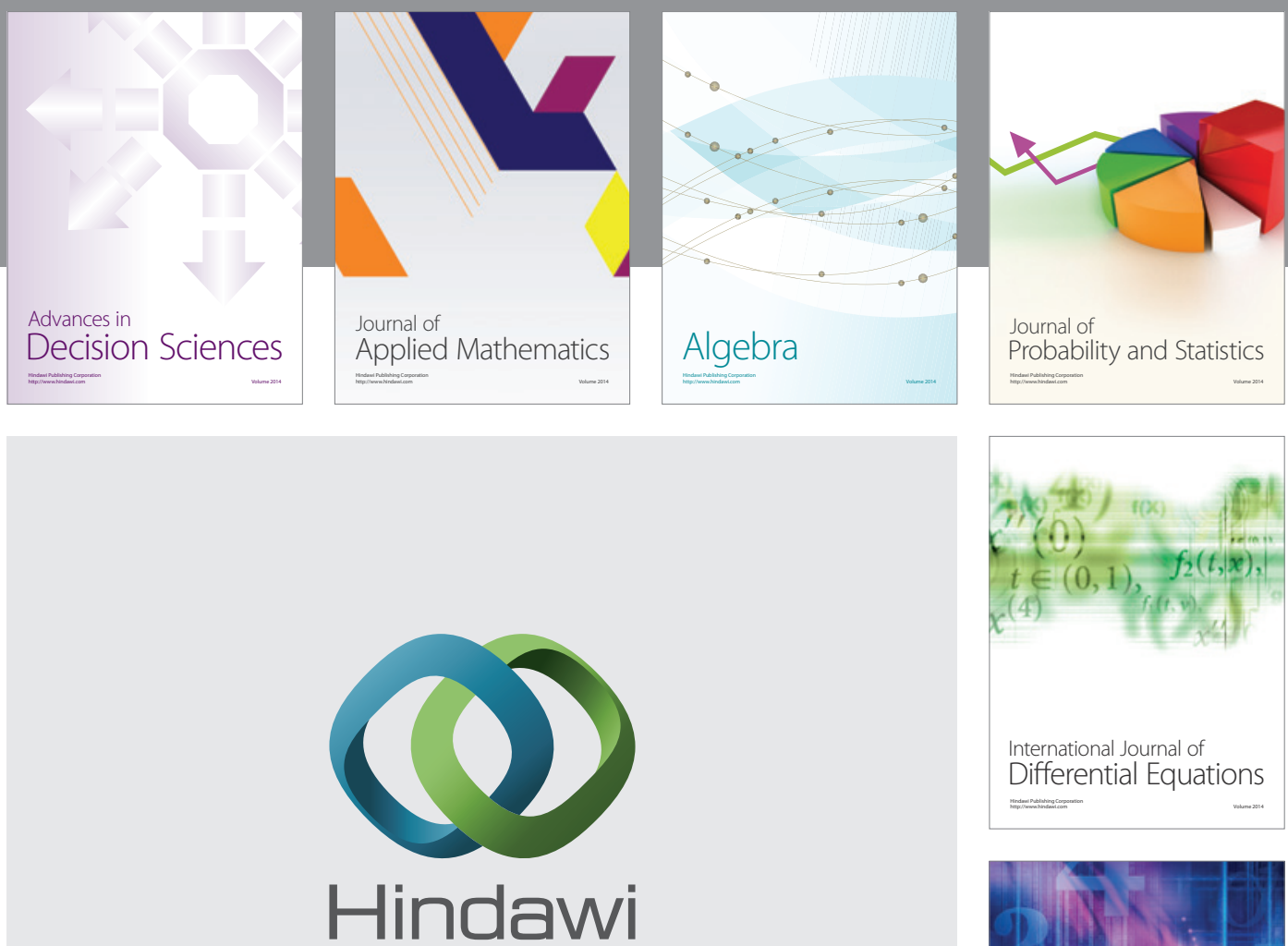

Submit your manuscripts at http://www.hindawi.com
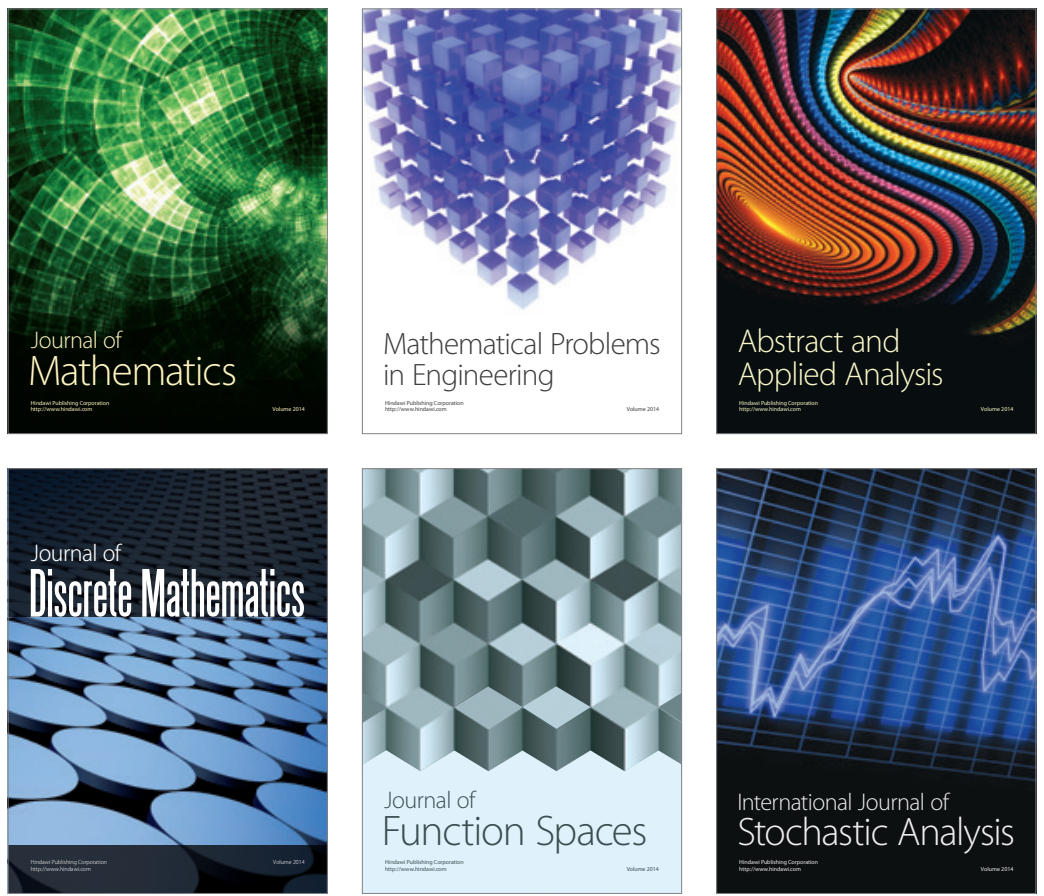

Journal of

Function Spaces

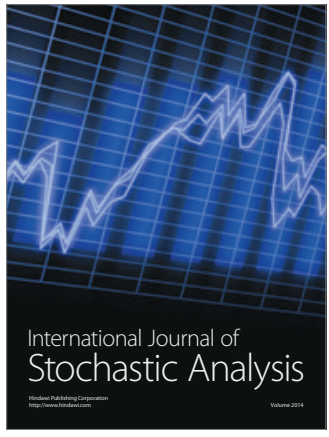

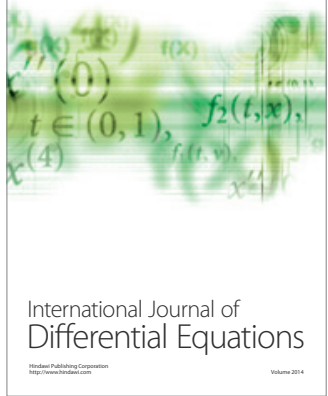
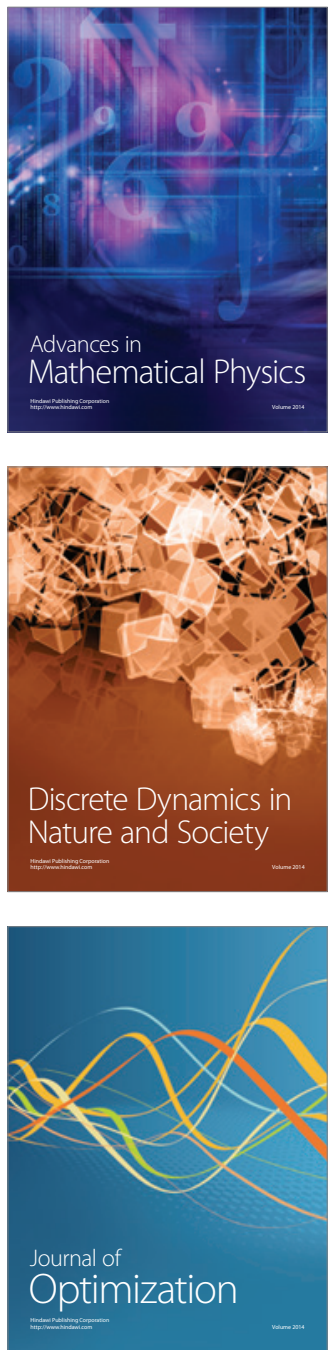